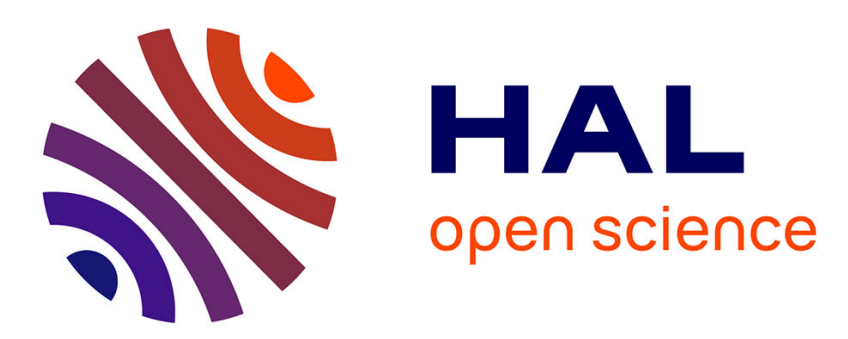

\title{
Structural and mechanical properties of single-wall carbon nanotube fibers
}

\author{
V. Pichot, S. Badaire, P.A. Albouy, C. Zakri, P. Poulin, P. Launois
}

\section{To cite this version:}

V. Pichot, S. Badaire, P.A. Albouy, C. Zakri, P. Poulin, et al.. Structural and mechanical properties of single-wall carbon nanotube fibers. Physical Review B: Condensed Matter and Materials Physics (1998-2015), 2006, 74, pp.245416. 10.1103/PhysRevB.74.245416 . hal-00131243

\section{HAL Id: hal-00131243 \\ https://hal.science/hal-00131243}

Submitted on 2 Sep 2021

HAL is a multi-disciplinary open access archive for the deposit and dissemination of scientific research documents, whether they are published or not. The documents may come from teaching and research institutions in France or abroad, or from public or private research centers.
L'archive ouverte pluridisciplinaire HAL, est destinée au dépôt et à la diffusion de documents scientifiques de niveau recherche, publiés ou non, émanant des établissements d'enseignement et de recherche français ou étrangers, des laboratoires publics ou privés. 


\title{
Structural and mechanical properties of single-wall carbon nanotube fibers
}

\author{
V. Pichot, ${ }^{1}$ S. Badaire, ${ }^{2}$ P. A. Albouy, ${ }^{1}$ C. Zakri, ${ }^{2}$ P. Poulin,${ }^{2}$ and P. Launois ${ }^{1, *}$ \\ ${ }^{1}$ Laboratoire de Physique des Solides (UMR CNRS 8502), bât 510, Université Paris Sud, 91405 Orsay, France \\ ${ }^{2}$ Centre de Recherche Paul Pascal, CNRS, Université Bordeaux I, avenue Schweitzer, 33600 Pessac, France
}

(Received 17 July 2006; revised manuscript received 28 August 2006; published 13 December 2006)

\begin{abstract}
We report quantitative experimental study correlating the structure and mechanical properties of fibers made from single-walled carbon nanotubes (SWNTs) and polyvinyl alcohol (PVA). A post-synthesis solvent drawing treatment is used to vary nanotube alignment, whose detailed understanding is a prerequisite for fiber development. Quantitative analysis of nanotube alignment within the fibers with different draw ratios is performed using x-ray scattering. The method is described in detail, and we also show that the improvement of nanotube alignment with draw ratio can be understood within a model of induced orientation at constant volume. Young's modulus and tensile strength increase with nanotube alignment. This is modeled using continuum mechanics in qualitative agreement with experiment, however quantitative differences show that nanotube alignment is not the only parameter controlling the fiber mechanical properties. We suggest that interaction between the SWNTs and PVA chains should also play a significant role.
\end{abstract}

DOI: 10.1103/PhysRevB.74.245416 PACS number(s): 61.46.Fg, 61.10.Eq, 62.25.+g, 68.05.Cf

\section{INTRODUCTION}

Individual carbon nanotubes possess great mechanical properties $^{1}$ that make them interesting components in macroscopic materials. For instance, Dalton et al. ${ }^{2}$ and Miaudet et al. $^{3}$ recently reported very high toughness in fibers made of nanotubes and polyvinyl alcohol (PVA). Applications in bulletproof vests, protective textiles or helmets, could be considered. Other potential applications of nanotube composite fibers in textiles, as distributed sensors, electronic interconnects, electromagnetic shielding, antennas, and batteries, are discussed in Ref. 4.

Nanotubes being highly anisotropic objects, the physical properties of nanotube-based materials depend on their alignment. ${ }^{5-8}$ In the present paper, we study correlation between nanotube alignment and mechanical properties of single-wall carbon nanotube (SWNT)-PVA fibers obtained by a wet-spinning process. ${ }^{9}$ Nanotube alignment can be improved by stretching the fibers. ${ }^{5}$ It is studied by $\mathrm{x}$-ray scattering. A model is presented allowing one to obtain the distribution of orientations in real space from the measurements in reciprocal space. The improvement of nanotube alignment as a function of the fiber draw ratio is well explained within the scope of an affine model of induced orientation at constant volume. Measurements of Young modulus and tensile strain as a function of the draw ratio are also shown. Both quantities are found to increase with nanotube alignment. The improvement of the Young modulus as a function of the nanotube alignment is discussed within continuum mechanics.

\section{EXPERIMENTAL}

The spinning method used for obtaining the SWNT-PVA fibers is detailed by Vigolo et al. and Poulin et al. in Refs. 9 and 10. The present fibers are spun from HiPCO nanotubes ${ }^{10}$ purchased from Carbon Nanotechnologies Inc. (batch CM26). An aqueous dispersion consisting of $0.3 \mathrm{wt}$ \% carbon nanotubes and $1.2 \mathrm{wt}$ \% sodium dodecyl sulfate (SDS) ionic surfactant is prepared. It is sonicated to achieve a good homogenization. The dispersion is then injected at $50 \mathrm{ml} / \mathrm{h}$ through a conical tip in the coflowing stream of a rotating bath $(100 \mathrm{rpm})$ of a polymer solution with $5 \mathrm{wt} \%$ PVA. A SWNT-PVA ribbon forms at the end of the tip and is later washed 3 times in pure water to remove part of the excess of PVA. The ribbon is then vertically drawn out of the solution and collapses in a long fiber, with typical diameter of about $30 \mu \mathrm{m}$. Thermogravimetric analysis shows that the relative PVA/NT weight ratio is about $50 / 50 \%$. To improve the alignment of the nanotubes, a stretching procedure ${ }^{5}$ is applied to the as-cast fibers. They are first rewetted in a $50 / 50 \mathrm{vol} \%$ mixture of water and acetone, then loaded with a given weight and finally dried under a tensile load. The corresponding elongation was determined for each given weight. The draw ratio of the fibers is defined by this equation

$$
\frac{\Delta L}{L_{0}}=\frac{L-L_{0}}{L_{0}},
$$

where $L_{0}$ and $L$ are respectively the fiber length before and after stretching ( $0 \%$ corresponds to the raw fiber). Draw ratios of the studied fibers are given in Table I.

$\mathrm{X}$-ray scattering experiments were performed on single fibers, using synchrotron radiation at LURE, France (beam line D43). To avoid background due to the fluorescence from iron catalyst used in the HiPCO process, ${ }^{11}$ experiments were performed at an energy below the $K$ absorption edge of iron, corresponding to a wavelength of $1.795 \AA$. The wavelength was selected using the (111) reflection of a curved germanium monochromator. Experiments were performed in transmission, under vacuum to avoid scattering by air, as detailed in Ref. 12. Diffraction patterns were recorded on planar Imaging Plates.

Mechanical properties were investigated by using a traction machine. The Young modulus and the tensile strength of each fiber in Table I were determined from the traction curves. The stress is calculated by dividing the applied force 
TABLE I. This table summarizes the draw ratios of the studied fibers, values of the half width at half maximum $w_{r}$ of the Gaussian orientational distribution of the nanotubes and the PVA chains, values of $r$ the ratio between the integrated intensity of the PVA signal measured along the direction corresponding to $\tau=0^{\circ}$ and the one corresponding to $\tau=90^{\circ}$.

\begin{tabular}{ccccc}
\hline \hline Fibers & Draw ratio & $w_{r}(\mathrm{NT})\left(^{\circ}\right)$ & $w_{r}(\mathrm{PVA})\left(^{\circ}\right)$ & $r$ \\
\hline Hi0 & $0 \%$ & 25 & 25 & 0.62 \\
Hi1 & $23 \%$ & 22 & 20.5 & 0.4 \\
Hi2 & $37 \%$ & 18 & 19 & 0.38 \\
Hi3 & $43 \%$ & 15 & 16 & 0.26 \\
Hi4 & $56 \%$ & 14 & 15.5 & 0.26 \\
Hi5 & $71 \%$ & 14.5 & 15.5 & 0.21 \\
\hline \hline
\end{tabular}

by the fiber section. The initial fiber section can be measured by Scanning Electron Microscopy. A constant volume hypothesis is applied to calculate the sections for different elongations during the traction experiment.

\section{RESULTS}

\section{A. Nanotubes alignment from $x$-ray scattering experiments}

\section{Experimental results}

A typical diffraction pattern from a HiPCO fiber is shown in Fig. 1. As discussed in Ref. 12, it consists of concentric rings, whose intensities are modulated angularly, corresponding to diffraction signals from the different components of the fiber, such as the PVA and nanotubes. A broad diffuse peak (black arrow in Fig. 1) at about $1.4 \AA^{-1}$ is attributed to the PVA. It corresponds to interchain interferences in the amorphous phase of the PVA. ${ }^{12,13}$ The nanotube signal is measurable below $1 \AA^{-1}$. It decreases with increasing wave vector and presents two modulations around 0.5 and $0.8 \AA^{-1}$,

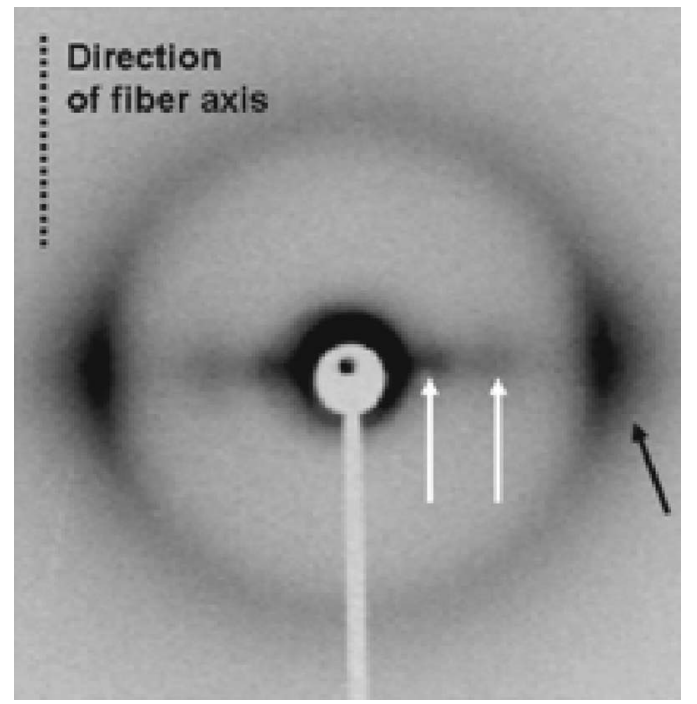

FIG. 1. Diffraction pattern obtained from a HiPCO fiber. The white and black arrows point the signal coming from the nanotubes and the PVA chains, respectively. in good agreement with diffraction data obtained on other HiPCO fibers in Ref. 7.

Preferential orientations of nanotubes and PVA chains in the fiber lead to modulations in the azimuthal intensity distributions. The intensity maxima are perpendicular to the fiber axis, which shows that nanotubes and PVA chains are preferentially aligned along this axis.,12 Intensities are scanned on Image Plates for constant wave vector values below $1 \AA^{-1}$, corresponding to the nanotube signal (similar results are found for different wave vectors below $1 \AA^{-1}$ ), and for wave vector $\mathrm{Q} \sim 1.4 \AA^{-1}$, corresponding to the PVA signal. They are reported as a function of the azimuthal angle $\tau$ in Fig. 2. Absorption or polarization corrections are negligible. Good fitting of the curves is obtained using Gaussian distributions. Half widths at half maximum $\left(w_{r}\right)$ of the Gaussian distributions are reported in Table I. They are roughly the same for the nanotubes and the PVA signals and decrease for increasing draw ratios.

The similarity in the angular dependences of nanotubes and PVA signals may appear surprising since PVA is a flexible polymer, the persistence length of which is about $9 \AA$. It shows that the orientation of PVA chains is templated by SWNTs. Indeed, a similar result has been obtained recently concerning the orientation of PVA crystallites in presence of SWNTs (see Ref. 14).

Radial scans performed along two perpendicular directions of the diffraction pattern allow one to measure nanotube and PVA signals corresponding to $\tau=0^{\circ}$, along the fiber axis and to $\tau=90^{\circ}$, perpendicular to it (see e.g. Fig. 4 in Ref. 12). Nonzero modulations of the nanotube or PVA signals are measured for $\tau=0^{\circ}$, along the fiber axis, while Gaussian functions determined above do not contribute any more at this value. One thus considers additional intensity contributions which are constant as a function of the azimuthal angle, corresponding to nonoriented nanotubes or PVA chains. As in Refs. 7 and 12, we will use a two-phase model to account for the fiber texture, with a fraction of nanotubes (or PVA chains) exhibiting preferential orientation along the fiber axis and a fraction of them exhibiting random orientations. The ratio between oriented and nonoriented fractions of nanotubes or PVA chains can be deduced from the ratio between signals at $\tau=0$ and $90^{\circ}$. Note that the $Q$ modulations of the nanotube signal, around 0.5 and $0.8 \AA^{-1}$, are much weaker than that of the PVA signal and cannot be quantified with good accuracy. But previous data obtained on fibers synthesized by using nanotubes produced by electric arc, ${ }^{12}$ where the signal coming from the nanotubes is more modulated (because of the narrower distribution of the nanotube diameters and bundles size) gave the same ratios for nanotube and PVA signals. This result, together with our above conclusion that SWNTs template the orientation of PVA, leads us to assume that the amount of nonoriented nanotubes is the same as PVA. Nanotubes and PVA are assembled in a very intricate network ${ }^{5}$ and would have the same orientational characteristics. Numerical values of the ratio $r$ between the integrated intensity (in wave vector $Q$ ) of the PVA signal along the direction corresponding to $\tau=0^{\circ}$ and the one corresponding to $\tau=90^{\circ}$ are reported in Table I.

\section{Analysis}

We explain here how one can rigorously deduce the orientation distribution function of nanotubes (PVA) from the 


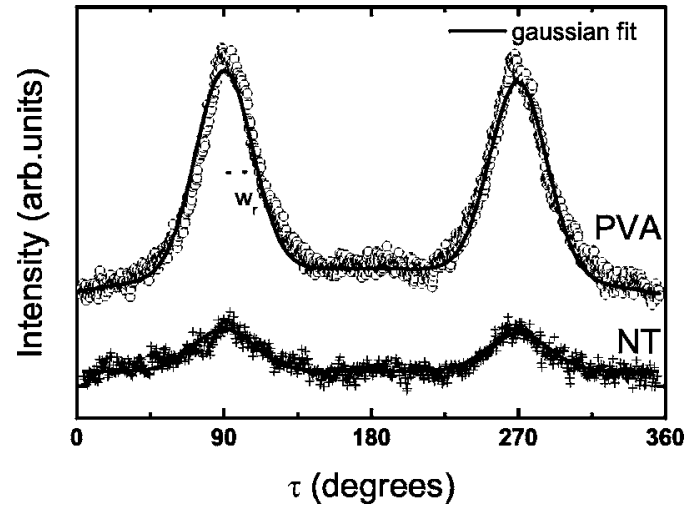

FIG. 2. Azimuthal intensities distribution at wave vectors below $1 \AA^{-1}$ (NT signal) and at $1.4 \AA^{-1}$ (PVA signal).

data obtained in the reciprocal space. To our knowledge, this has not been done in previous publications on nanotube orientation in composites.

In the two phase model, the distribution of orientations is defined by

$$
p(\theta)=\left[p_{1}(\theta)+f p_{2}(\theta)\right] /(1+f),
$$

where $\theta$ is the angle between a nanotube axis (PVA chain) and the fiber axis (Fig. 10). The orientation of the nanotube axis (PVA chain axis) with that of the fiber is also defined by a second angle $\varphi$ in the plane perpendicular to the fiber axis. However, the fiber being axially symmetric, the distribution has cylindrical symmetry and it does not depend on $\varphi$. The distribution $p_{1}(\theta)$ corresponds to the fraction of oriented nanotubes and $p_{2}(\theta)=1 /(4 \pi)$ corresponds to the nonoriented nanotubes. Both distributions are normalized to 1 $\left(\int_{0}^{2 \pi} d \varphi \int_{0}^{\pi} d \theta \sin (\theta) p_{i}(\theta)=1\right)$. The parameter " $f$ " gives the ratio between nonoriented and oriented fractions of nanotubes (PVA chains). Note that the same formalism being used for nanotubes and PVA chains, one will no more mention explicitly PVA chains in the following explanations.

We demonstrate in the Appendix a key relationship between the measured intensity on a planar detector and the orientation distribution function. ${ }^{15}$ It is

$$
I(\tau) \propto \int_{0}^{\pi / 2} p\left[\arccos \left\{\cos (\xi) \sin \left(\arccos \left[\cos \left(\theta_{B}\right) \cos (\tau)\right]\right)\right\}\right] d \xi .
$$

In this equation, $\theta_{B}$ is the Bragg angle, satisfying the relation $Q=4 \pi \sin \left(\theta_{B}\right) / \lambda$, and $\tau$ is the azimuthal angle on the detec-

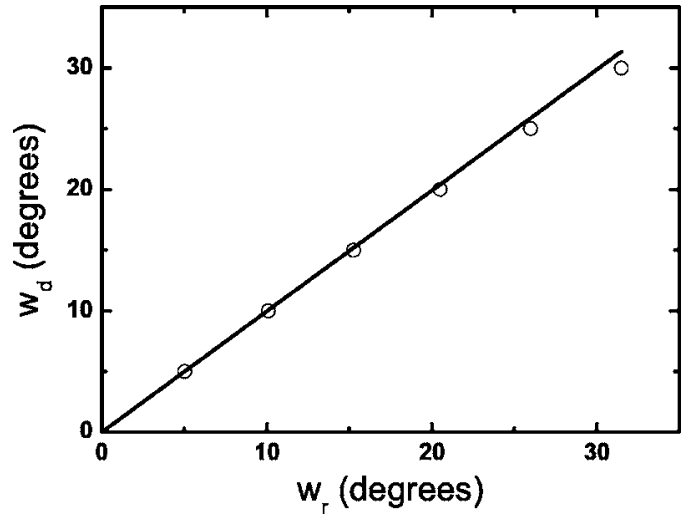

FIG. 3. Half width at half maximum of a Gaussian distribution of orientations in direct space $w_{d}$ as a function of the one of the corresponding Gaussian distribution in intensity on a planar detector in reciprocal space $w_{r}$, for $\theta_{B}=5.9^{\circ}$ (corresponding to $Q$ $\left.\sim 0.7 \AA^{-1}\right)$. The straight line has equation $w_{d}=w_{r} \cos \left(\theta_{B}\right)$.

tor. The only hypothesis made is that scattering is located in the plane perpendicular to the nanotube axis. It is valid for the diffraction peaks considered here: for small wave vectors the nanotube scattering corresponds to scattering in the plane $1=0$ and the PVA peak at $Q \sim 1.4 \AA^{-1}$ corresponds to interchain distances perpendicular to the long chains.

In Sec. III A 1, the measured intensity has been fitted with a Gaussian distribution plus a constant term. For a given wave vector, it writes

$$
I(\tau)=I_{1}(\tau)+I_{2}(\tau)
$$

with $I_{1}(\tau)=\alpha(1-r) \exp \left\{(-\ln 2)\left[(\tau-\pi / 2) / w_{r}\right]^{2}\right\}$ and

$$
I_{2}(\tau)=\alpha r
$$

$(0 \leqslant \tau \leqslant \pi), \alpha$ is a proportionality term that depends on wave vector but not on azimuthal angle.

We have considered different orientation functions, calculated numerically their integrals in Eq. (2) and fitted the obtained intensity curves. We have shown that, except for too large Gaussian widths, a Gaussian orientation distribution function gives an intensity curve that is well fitted by a Gaussian function also

$$
\int_{0}^{\pi / 2} \exp \left((-\ln 2)\left[\arccos \left\{\cos (\xi) \sin \left(\arccos \left[\cos \left(\theta_{B}\right) \cos (\tau)\right]\right)\right\} / w_{d}\right]^{2}\right) d \xi \propto \exp \left\{(-\ln 2)\left[(\tau-\pi / 2) / w_{r}\right]^{2}\right\}
$$

The relationship between Gaussian widths of the orientation distribution function and that of the calculated intensity $\left(w_{d}\right.$ and $w_{r}$ ) is illustrated in Fig. 3. For small values of $\theta_{B}$, one finds $w_{d}=w_{r} \cos \left(\theta_{B}\right)$; this result can be easily deduced from Eq. (2) using expansion in series up to second order in $x$. For larger widths, the gap between $w_{d}$ and $w_{r}$ slightly increases. 
Using this result, Table I and Fig. 3, one concludes that the distribution function $p_{1}$ of oriented nanotubes (PVA chains) is a Gaussian function and we obtain its width $w_{d}$. Results for the different fibers studied, calculated from the mean value of $w_{r}$ for nanotubes and for PVA, are drawn in Fig. 4 (within error bars, PVA and nanotubes have the same $w_{d}$, the role of the $\cos \left(\theta_{B}\right)$ term is minor for the relatively small wave vector values considered).

Equations (1), (2), and (3) give

$$
(1-r) \exp \left\{(-\ln 2)\left[(\tau-\pi / 2) / w_{r}\right]^{2}\right\}+r=A \frac{\int_{0}^{\pi / 2} p_{1}\left[\arccos \left\{\cos (\xi) \sin \left(\arccos \left[\cos \left(\theta_{B}\right) \cos (\tau)\right]\right)\right\}\right] d \xi+\frac{f}{8}}{1+f}
$$

with

$$
p_{1}(\theta)=\frac{\exp \left[(-\ln 2)\left(\theta / w_{d}\right)^{2}\right]}{4 \pi \int_{0}^{\pi / 2} \exp \left[(-\ln 2)\left(\theta / w_{d}\right)^{2}\right] \sin \theta d \theta},
$$

" $A$ " being a constant.

Using Eq. (4) for $\tau=0$ and $\pi / 2$, one gets a system of two equations where from one obtains that the ratio between the total number of nonoriented nanotubes (PVA chains) and that of oriented one writes

$$
f=8 \frac{r}{(1-r)} \int_{0}^{\pi / 2} p_{1}(\xi) d \xi
$$

Values of $f$ for the different fibers studied are drawn in Fig. 4.

In summary, both $w_{d}$ and $f$ values decrease for increasing drawing ratios: these results clearly show that nanotubes and PVA chains get better aligned in drawn fibers. The best value found for $w_{d}$ is around $15^{\circ}$. It is much better than the first reported results on electric-arc nanotube fibers $\left(w_{r} \sim 37^{\circ}\right)$ and similar to results obtained on the same kind of drawn fibers. ${ }^{16}$ With respect to the literature, nanotubes in our fibers

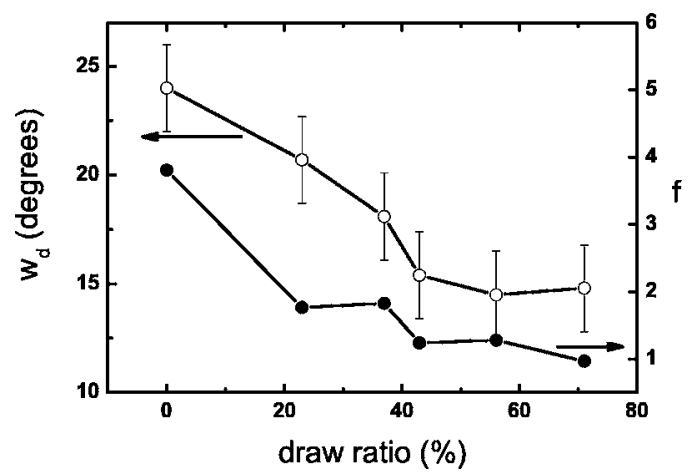

FIG. 4. Half width at half maximum $w_{d}$ of the Gaussian distribution function describing the alignment of the oriented fraction of nanotubes (PVA chains) and fraction of nonoriented nanotubes (PVA chains) with respect to the oriented one, as functions of fiber draw ratios. present a good alignment. Ericson et al. ${ }^{17}$ and Zhou et al. ${ }^{7}$ reported values of $w_{r}$ of about $16^{\circ}$ in fibers realized from HiPCO nanotubes extruded from super-acid suspensions. The smallest width reported in the literature is $\sim 9^{\circ},{ }^{3}$ corresponding to fibers treated in a different way than in this paper, by high temperature stretching. Fraction of nonoriented nanotubes can also be deduced from Raman measurements ${ }^{7,18}$ and best reported values are around 1 , as in our study.

\section{B. Mechanical properties}

Mechanical properties of the fibers have been studied by using a stretching machine Zwick 2.5/TN1S. An example of traction curve obtained from a HiPCO fiber is given in Fig. 5.

Figure 6 shows that the Young modulus and the tensile strength increase with the drawing ratio of the fiber, that is for improved nanotube alignments. The strain to failure, which is found to vary between 3 and $12 \%$ in our measurements on different fibers, is not given here, because its values are not reproducible. Indeed, it is very dependent of the structural defaults. Young modulus can reach $22 \mathrm{GPa}$, and tensile strength $170 \mathrm{MPa}$. These values are not the best obtained for a nanotube fiber. ${ }^{2,3}$ But the interest of the present results is that they are the only quantitative results which allow one to analyze the evolution of the mechanical properties of the fibers with the alignment of the nanotubes in the fibers.

\section{DISCUSSION}

\section{A. Alignment of the nanotubes and PVA chains}

We have shown that the width $w_{d}$ of the Gaussian orientation distribution of the nanotubes (PVA chains) and the amount $f$ of the nonoriented nanotubes (PVA chains) decrease when the draw ratios of fibers increase.

To go further, we describe the evolution of these two quantities by using an induced ordering affine model. ${ }^{14,19}$ Scanning electron microscopy measurements of the evolution of the cross section area $S$ of fibers treated by solvent drawing, as the function of the draw ratios of the fibers, indicate that under this treatment, the volume $S L$ of the fibers 


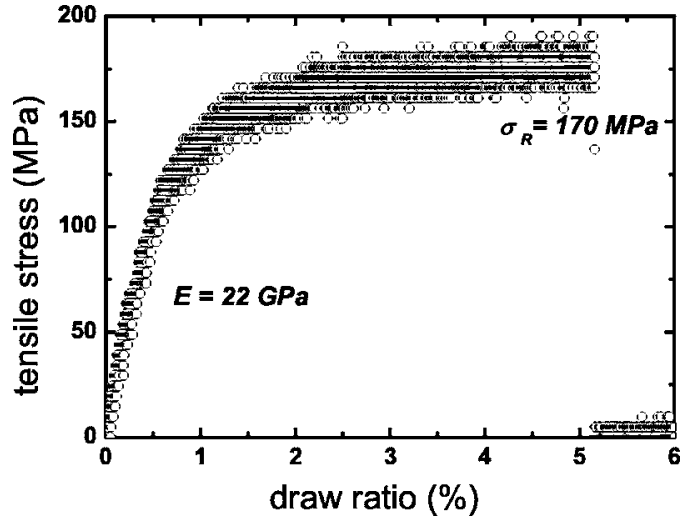

FIG. 5. Stress versus strain curve for fiber Hi5 (Young modulus $E=22 \mathrm{GPa}$, tensile strength $\sigma_{R}=170 \mathrm{MPa}$ ).

remains constant. Within a constant volume hypothesis, the tensor of deformation of the strained fiber can be written as follows:

$$
\Lambda=\left[\begin{array}{ccc}
1 / \sqrt{\lambda} & 0 & 0 \\
0 & 1 / \sqrt{\lambda} & 0 \\
0 & 0 & \lambda
\end{array}\right],
$$

where $\lambda$ is defined by: $\lambda=L_{f i n} / L_{i n i}=1+\Delta L / L$. After stretching, the fiber dimensions along and perpendicular to the stretching axis $\left(L z^{\prime}\right.$ and $\left.L^{\prime}{ }_{\perp}\right)$ are related to initial dimensions ( $L z$ and $L_{\perp}$ ) by: $L_{z}^{\prime}=\lambda L_{z}$ and $L^{\prime}=1 / \sqrt{\lambda} L_{\perp}$. The angle $\theta$ between a nanotube (or a PVA chain) and the fiber axis is changed in $\theta^{\prime}$, with:

$$
\operatorname{tg}\left(\theta^{\prime}\right)=L_{\perp}^{\prime} / L_{z}^{\prime}=1 / \lambda^{3 / 2} \operatorname{tg}(\theta)
$$

(see Fig. 7). The orientation distribution in the stretch fiber can be calculated converting the variable $\theta$ into $\theta^{\prime}$.

Equation (6) allows one to calculate

$$
\sin (\theta) d \theta \rightarrow \frac{\lambda^{3}}{\left[\cos ^{2}\left(\theta^{\prime}\right)+\lambda^{3} \sin ^{2}\left(\theta^{\prime}\right)\right]^{3 / 2}} \sin \left(\theta^{\prime}\right) d \theta^{\prime} .
$$

Thus, the new orientation distribution $p^{\prime}\left(\theta^{\prime}\right)$ is

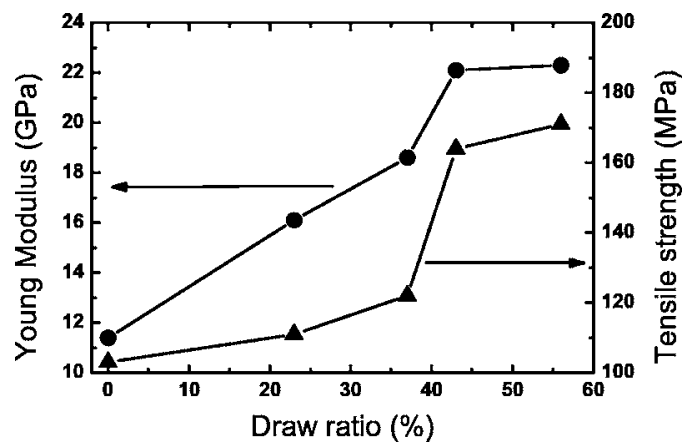

FIG. 6. Young modulus and tensile strength for fibers with different draw ratios.

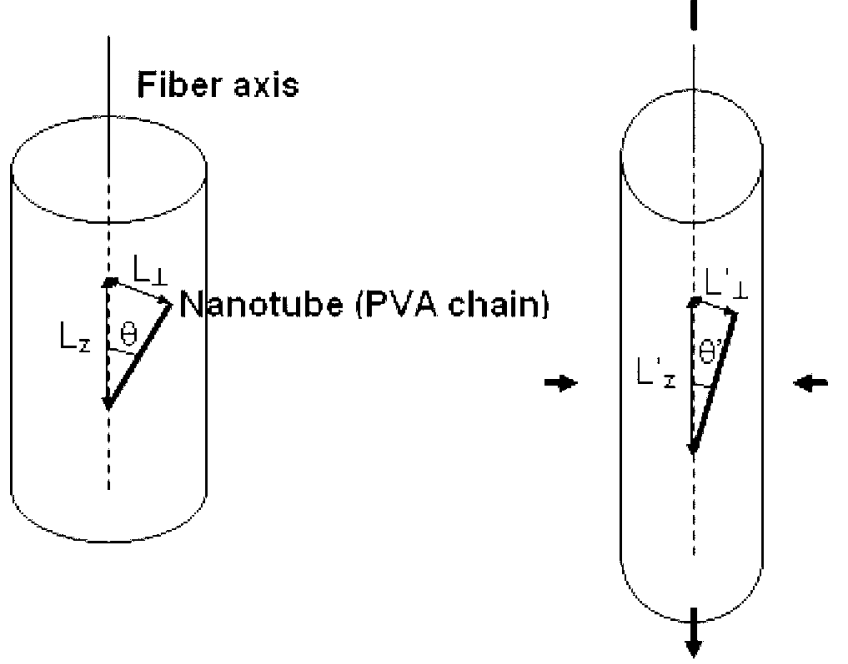

FIG. 7. Scheme of an affine extension (left: as-cast fiber, right: stretched fiber).

$$
p^{\prime}\left(\theta^{\prime}\right)=\frac{\lambda^{3}}{\left[\cos ^{2}\left(\theta^{\prime}\right)+\lambda^{3} \sin ^{2}\left(\theta^{\prime}\right)\right]^{3 / 2}} p\left\{\arctan \left[\lambda^{3 / 2} \operatorname{tg}\left(\theta^{\prime}\right)\right]\right\} .
$$

Within the two-phase model used above, one finally obtains

$$
\begin{aligned}
p^{\prime}\left(\theta^{\prime}\right)= & \frac{\lambda^{3}}{\left[\cos ^{2}\left(\theta^{\prime}\right)+\lambda^{3} \sin ^{2}\left(\theta^{\prime}\right)\right]^{3 / 2}}\left(p_{1}\left\{\arctan \left[\lambda^{3 / 2} \operatorname{tg}\left(\theta^{\prime}\right)\right]\right\}\right. \\
& \left.+f p_{2}\right) /(1+f) .
\end{aligned}
$$
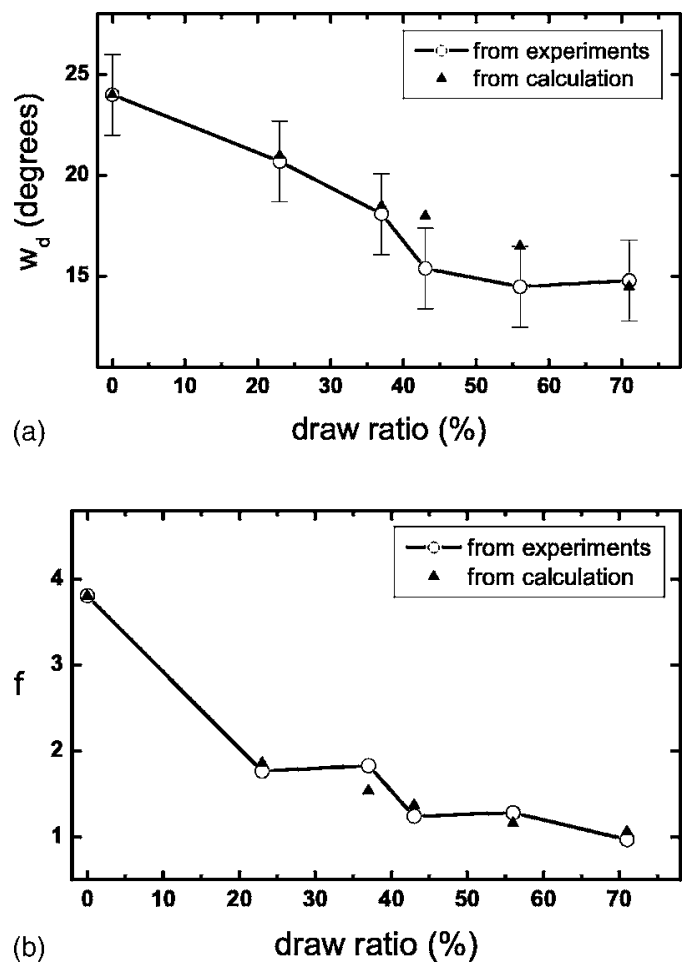

FIG. 8. Evolution of $w_{d}$ and $f$ as a function of the draw ratio of the nanotubes fibers, open circles are experimental data and filled triangles correspond to calculations within the scope of an affine model of induced ordering. 
From the alignment values deduced from experimental data on fiber HiO, one can calculate for each value of the draw ratios (corresponding to different values of $\lambda$ ) the function $p^{\prime}\left(\theta^{\prime}\right)$. It can then be fitted by using a constant and a Gaussian function, which gives: $p^{\prime}\left(\theta^{\prime}\right)=\left[p^{\prime}{ }_{1}\left(\theta^{\prime}\right)\right.$ $\left.+f^{\prime} p^{\prime}{ }_{2}\left(\theta^{\prime}\right)\right] /\left(1+f^{\prime}\right)$ where $p^{\prime}{ }_{1}$ et $p^{\prime}{ }_{2}$ are correctly normalized $\left[\iint p^{\prime}{ }_{i}\left(\theta^{\prime}\right) \sin \left(\theta^{\prime}\right) d \theta^{\prime} d \varphi^{\prime}=1\right] \cdot p^{\prime}{ }_{1}$ is described by its half width at half maximum $w_{d}^{\prime}$ and $f^{\prime}$ is the amount of nonoriented nanotubes. The calculated values with the experimental ones are drawn in Fig. 8. The agreement between experiments and calculations is good.

The mechanism involved in the solvent drawing postsynthesis treatment is thus found to be a simple reorientation process of both nanotubes and PVA chains under affine in- compressible extension; no sliding phenomena occur in the studied range of draw ratios. Let us underline here that understanding of the phenomena in play to improve nanotube alignment in composites should be of great help for future developments of their applications.

\section{B. Mechanical properties}

We have shown that the mechanical properties of the fibers are improved with the alignment of the nanotubes. Liu and $\operatorname{Kumar}^{20}$ predicted using continuum mechanics an increase of the Young modulus with the alignment of the nanotubes. We apply their model to our experimental results. The Young modulus in a sample of oriented nanotubes in a fiber can be written

$$
E=\frac{0.5}{\frac{1}{E_{2}}+\left(\frac{1}{G_{12}}-\frac{2 v_{12}}{E_{1}}-\frac{2}{E_{2}}\right)\left\langle\cos ^{2}(\theta)\right\rangle+\left(\frac{1}{E_{1}}+\frac{1}{E_{2}}-\frac{1}{G_{12}}+\frac{2 v_{12}}{E_{1}}\right)\left\langle\cos ^{4}(\theta)\right\rangle}
$$

This formula is the one given in Ref. 9, except for the factor 0.5 introduced here take into account the nanotubes density in the fibers (50 wt. \%). In Eq. (8), $E_{1}, E_{2}, G_{12}$, and $v_{12}$ are respectively the longitudinal modulus, transverse modulus, in-plane shear modulus and the Poisson's ratio of a nanotubes bundle. Average values of $\left\langle\cos ^{2}(\theta)\right\rangle$ and $\left\langle\cos ^{4}(\theta)\right\rangle$ are calculated from the orientational distribution of the nanotubes i.e. from the data from Fig. 8 for Hi1-Hi5 fibers. As Liu and Kumar, ${ }^{9}$ we take $E_{1}=640 \mathrm{GPa}, E_{2}=15 \mathrm{GPa}$, and $v_{12}=0.17$ (which are approximately the calculated values of Popov et al. ${ }^{21}$ ) and we fit the $G_{12}$ value to account for experimental results ( $G_{12}$ value depends on bundle sizes). We obtain $G_{12}=15 \mathrm{GPa}$ which is a reasonable value with respect to the results obtained for small diameter bundles by Salvetat et al. ${ }^{22}$ bundles of HiPCO nanotubes being usually rather small. Calculated and measured values of the Young modulus are reported in Fig. 9. Agreement between the measurements and calculation is only qualitative as the variation of the calculated Young modulus is too weak, but one rightly finds, as in Ref. 20, an increase of the Young modulus with improvement of the nanotubes alignment. Within the scope of the above model, for perfect alignment of the nanotubes $\left(\left\langle\cos ^{2}(\theta)\right\rangle=1\right.$ and $\left.\left\langle\cos ^{4}(\theta)\right\rangle=1\right)$, Young modulus would be equal to $E_{1} / 2=320 \mathrm{GPa}$, to be compared with $22 \mathrm{GPa}$ for fiber Hi5 in our study: progresses can thus still be expected with improved alignment of the nanotubes.

The model of Liu and $\mathrm{Kumar}^{20}$ only takes into account the nanotubes mechanical properties while it is applied here to a composite material made for $50 \mathrm{wt} . \%$ of PVA. The fact that agreement between calculations and experiments is qualitative but not quantitative shows that SWNT alignment is not the only parameter to control in order to improve the mechanical properties of the fibers. In the case of composites with a much smaller amount of nanotubes, Cadek, Coleman et al. ${ }^{23,24}$ have recently stressed the important role of the interface between PVA and nanotubes, where the stress transfer is maximized for crystalline PVA coating. The present case is different since it concerns composites with a large amount of nanotubes and because PVA is amorphous and not crystalline. However, our structural results, which show that the orientation of the PVA chains is templated by SWNTs, clearly point towards rather important interactions between nanotubes and amorphous PVA chains. One thus argues that the interface mechanical properties and/or the mechanical properties of templated PVA chains (which become rather rigid) also play a role in the mechanical properties of the fibers.

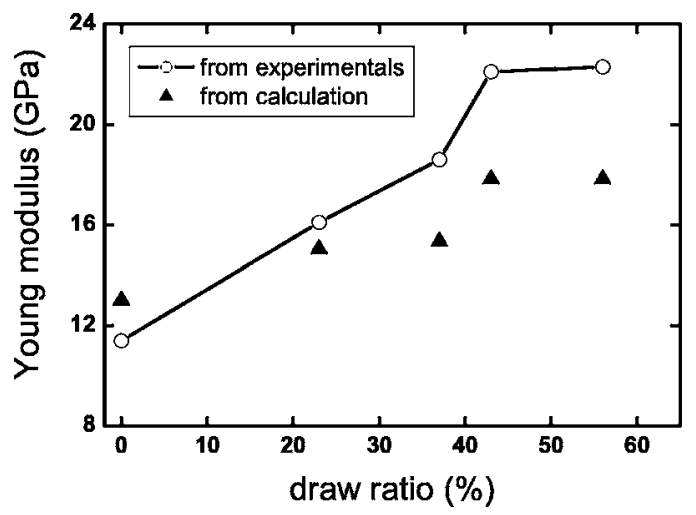

FIG. 9. Fibers Hi1-Hi5: measured Young modulus (open circles) and calculations (cross) as a function of the draw ratio of the fibers. 


\section{CONCLUSION}

In this paper, the degrees of alignment along the fibers axis of nanotubes and of PVA chains, whose orientation seems to be templated by nanotubes, was determined from $\mathrm{x}$-ray scattering experiments for different stretchings of the fibers. When the draw ratios of the fibers increase, the alignment of the nanotubes and PVA chains along the fiber axis becomes better. X-ray diffraction experiments allowed us to determine quantitatively the nanotube (PVA chain) alignment degree. An affine model of induced ordering at constant volume allows one to understand the improvement of nanotube (PVA chain) alignment for stretched fibers.

By coupling structural results with mechanical measurements on the fibers, one shows that the better the nanotube alignment along the fiber axis, the higher the tensile strength and the Young modulus. The mechanical characteristics reported here are not the best obtained for nanotube fibers, but they are the first ones to allow correlating structural measurements with mechanical properties on a macroscopic assembly of carbon nanotubes. Results are analyzed within the scope of a continuum mechanics model which indicates that much better values of Young modulus could be expected for very good alignments of the nanotubes along the fiber axis. Moreover, it is found that other parameters, such as adhesion between the matrix and the nanotubes and mechanical properties of the matrix itself, should be taken into account in the elaboration of resistive nanotube composite materials.

\section{ACKNOWLEDGMENTS}

Dominique Durand is acknowledged for her help in the synchrotron experiments at LURE. Helpful discussions with Georges Désarmot and Laurence Noirez are also acknowledged.

\section{APPENDIX: ANGULAR MODULATION OF THE EQUATORIAL INTENSITY DIFFRACTED BY NANOTUBES WITH PREFERRED ORIENTATION IN A FIBER}

Let us define a basis where the $z$ axis is the fiber axis. Orientation of the long axis of a nanotube and of a scattering vector $\vec{Q}$ in this basis are given by the spherical coordinates

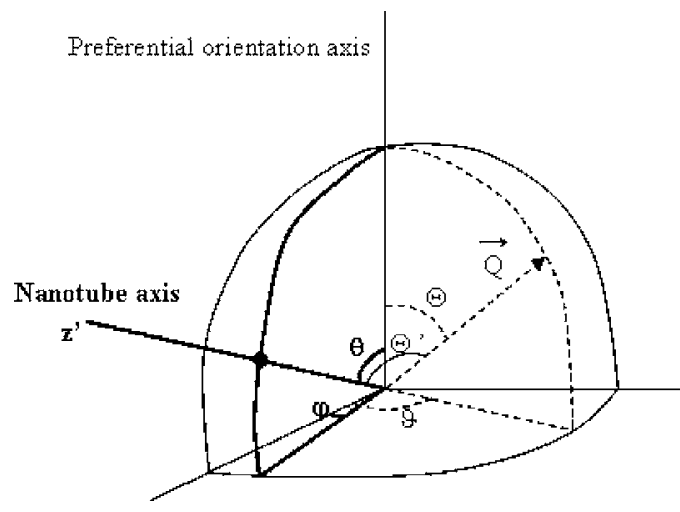

FIG. 10. Definition of angles used in the text.

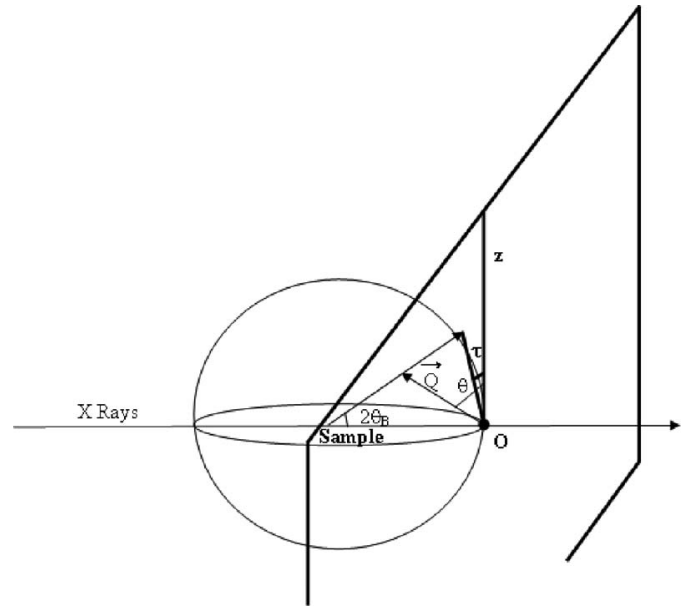

FIG. 11. Schematic drawing of the experimental configuration using the Ewald sphere representation. Since one is only interested here in angular quantities, to simplify the drawing, the detector is not drawn at distance $D$ from the sample but tangent to the Ewald sphere.

$(\theta, \varphi)$ and $(\Theta, \vartheta)$, respectively (Fig. 10). In the nanotube basis, where $z^{\prime}$ is the nanotube axis, orientation of the wave vector $\vec{Q}$ is given by $\left(\Theta^{\prime}, \vartheta^{\prime}\right)$. Due to axial symmetry of the fiber, the nanotubes orientation distribution $p(\theta)$ does not depend on $\varphi$.

Let us consider equatorial scattering coming from nanotubes having all possible orientations with respect to their long axis. It writes

$$
i \propto f\left[Q \sin \left(\Theta^{\prime}\right)\right] \delta\left[Q \cos \left(\Theta^{\prime}\right)\right],
$$

or equivalently

$$
i \propto \frac{1}{Q} f(Q) \delta\left[Q \cos \left(\Theta^{\prime}\right)\right],
$$

where $\delta$ is the Dirac distribution and where $f$ is a function depending on nanotubes diameters and on their possible organization in bundles. Using the spherical triangle relationship

$$
\cos \left(\Theta^{\prime}\right)=\cos (\theta) \cos (\Theta)+\sin (\theta) \sin (\Theta) \cos (\vartheta-\varphi),
$$

one gets $i \propto \delta[g(\varphi)] \quad$ with $g(\varphi)=\cos (\theta) \cos (\Theta)$ $+\sin (\theta) \sin (\Theta) \cos (\vartheta-\varphi)$.

The intensity on a pixel of the planar detector, situated on the circle corresponding to diffraction with angle $2 \theta_{B}$, varies with the angle $\tau$ of the projection of the scattering vector $\vec{Q}$ on the detector (Fig. 11). It is proportional to the sum of the intensities scattered by nanotubes with all orientations weighted by their orientational probability

$$
I(\tau) \propto \int_{0}^{\pi} d \theta \sin (\theta) f(\theta)\left[\int_{0}^{2 \pi} d \varphi \delta[g(\varphi)]\right] .
$$

Since $I(\pi-\tau)=I(\tau)$, we will consider here $\tau$ values between 0 and $\pi$. The integral over $\varphi$ can easily 
be calculated using the following relation: $\delta[g(\varphi)]$ $=\Sigma_{\varphi_{i} \text { verifying } g\left(\varphi_{i}\right)=0, g^{\prime}\left(\varphi_{i}\right) \neq 0} \frac{1}{\left|g^{\prime}\left(\varphi_{i}\right)\right|} \delta\left(\varphi-\varphi_{i}\right) . g\left(\varphi_{i}\right)=0$ is equal to zero for $\varphi_{i}$ such that $\cos \left(\vartheta-\varphi_{i}\right)=-\frac{\tan \left(\frac{\pi}{2}-\Theta\right)}{\tan \theta}$, which is only possible if $\frac{\pi}{2}-\Theta \leqslant \theta \leqslant \frac{\pi}{2}+\Theta$. In this case, $\left|g^{\prime}\left(\varphi_{i}\right)\right|$ $=\left|\sin \theta \sin \Theta \sin \left(\vartheta-\varphi_{i}\right)\right|=\sqrt{\sin ^{2} \theta-\cos ^{2} \Theta}$.

It follows that $I(\tau) \propto \int_{\pi / 2-\Theta}^{\pi / 2+\Theta} \frac{p(\theta) \sin (\theta) d \theta}{\sqrt{\sin ^{2}(\theta)-\cos ^{2}(\Theta)}}$

$\propto \int_{\pi / 2-\Theta}^{\pi / 2} \frac{p(\theta) \sin (\theta) d \theta}{\sqrt{\sin ^{2}(\theta)-\cos ^{2}(\Theta)}}$ (the integral between $\pi / 2-\Theta$ and $\pi / 2+\Theta$ is equal to two times the integral between $\pi / 2-\Theta$ and $\pi / 2$ ). Angles $\tau$ and $\Theta$ being related by equation $\cos (\Theta)=\cos (\tau) \cos \left(\theta_{B}\right), \quad$ one $\quad$ obtains $\quad I(\tau)$ $\sim \int_{\pi / 2-\arccos \left[\cos \left(\theta_{B}\right) \cos (\tau)\right]}^{\pi / 2} \frac{p(\theta) \sin (\theta) d \theta}{\sqrt{\sin ^{2}(\theta)-\cos ^{2}\left(\theta_{B}\right) \cos ^{2}(\tau)}}$. The denominator is zero for $\theta=\pi / 2-\arccos \left[\cos \left(\theta_{B}\right) \cos (\tau)\right]$. An easy-to-handle formulation of the integral is obtained with replacing variable $\theta$ by the variable $\xi$ defined by $\cos (\xi)$ $=\cos (\theta) / \sin \left\{\arccos \left[\cos \left(\theta_{B}\right) \cos (\tau)\right]\right\}$. One then gets Eq. (2) $I(\tau) \propto \int_{0}^{\pi / 2} p\left[\arccos \left\{\cos (\xi) \sin \left(\arccos \left[\cos \left(\theta_{B}\right) \cos (\tau)\right]\right)\right\}\right] d \xi$.
*Corresponding author. Email address: launois@1ps.u-psud.fr

${ }^{1}$ J.-P. Salvetat-Delmotte and A. Rubio, Carbon 40, 1729 (2002).

${ }^{2}$ A. B. Dalton, S. Collins, E. Munoz, J. M. Razal, V. H. Ebron, J. P. Ferraris, J. N. Coleman, B. G. Kim, and R. H. Baughman, Nature (London) 423, 703 (2003).

${ }^{3}$ P. Miaudet, S. Badaire, M. Maugey, A. Derré, V. Pichot, P. Launois, P. Poulin, and C. Zakri, Nano Lett. 5, 2212 (2005).

${ }^{4}$ A. B. Dalton, S. Collins, J. Razal, E. Munoz, V. H. Ebron, B. G. Kim, J. N. Coleman, J. P. Ferraris, and R. H. Baughman, J. Mater. Chem. 14, 1 (2004).

${ }^{5}$ B. Vigolo, M. Lucas, P. Launois, P. Bernier, and P. Poulin, Appl. Phys. Lett. 81, 1210 (2002).

${ }^{6}$ P. Launois and P. Poulin, Encyclopedia of Nanoscience and Nanotechnology, edited by H. S. Nalwa (American Scientific, Stevenson Ranch, CA, 2004), Vol. 4, p. 1.

${ }^{7}$ W. Zhou, J. Vavro, C. Guthy, K. I. Winey, J. E. Fischer, L. M. Ericson, S. Ramesh, R. Saini, V. A. Davis, C. Kittrell, M. Pasquali, R. H. Hauge, and R. E. Smalley, J. Appl. Phys. 95, 649 (2004).

${ }^{8}$ S. Badaire, V. Pichot, C. Zakri, P. Poulin, P. Launois, J. Vavro, C. Guthy, M. Chen, and J. E. Fischer, J. Appl. Phys. 96, 7509 (2004).

${ }^{9}$ B. Vigolo, A. Pénicaud, C. Coulon, C. Sauder, R. Pailler, C. Journet, P. Bernier, and P. Poulin, Science 290, 1331 (2000).

${ }^{10}$ P. Poulin, B. Vigolo, and P. Launois, Carbon 40, 1741 (2002).

${ }^{11}$ P. Nikolaev, J. Nanosci. Nanotechnol. 4, 307 (2004).

${ }^{12}$ P. Launois, A. Marucci, B. Vigolo, P. Bernier, A. Derré, and P. Poulin, J. Nanosci. Nanotechnol. 1, 125 (2001).

${ }^{13}$ P.-D. Hong and K. Miyasaki, Polymer 35, 1369 (1994).

${ }^{14}$ M. L. Minus, H. G. Chae, and S. Kumar, Polymer 47, 3705
(2006).

${ }^{15}$ O. Kratky, Kolloid-Z. 64, 213 (1933).

${ }^{16}$ M. Lucas, B. Vigolo, S. Badaire, D. Le Bolloc'h, A. Marucci, D. Durand, M. Hamilton, C. Zakri, P. Poulin, and P. Launois, in Proceedings of the XVIth International Winterschool on Electronic Properties of Novel Materials, Kirchberg, 2002, AIP Conference 633, edited by H. Kuzmany, J. Fink, M. Mehring and S. Roth, p. 579.

${ }^{17}$ L. M. Ericson, H. Fan, H. Peng, V. A. Davis, W. Zhou, J. Sulpizio, Y. Wang, R. Booker, J. Vavro, C. Guthy, A. N. G. Parra-Vasquez, M. J. Kim, S. Ramesh, R. K. Saini, C. Kittrell, G. Lavin, H. Schmidt, W. W. Adams, W. E. Billups, M. Pasquali, W.-F. Hwang, R. H. Hauge, J. E. Fischer, and R. E. Smalley, Science 305, 1447 (2004).

${ }^{18}$ E. Anglaret, A. Righi, J. L. Sauvajol, P. Bernier, B. Vigolo, and P. Poulin, Phys. Rev. B 65, 165426 (2002).

${ }^{19}$ S. V. Ahir and E. M. Terentjev, Nat. Mater. 4, 491 (2005).

${ }^{20}$ T. Liu and S. Kumar, Nano Lett. 3(5), 647 (2003).

${ }^{21}$ V. N. Popov, V. E. Van Doren, and M. Balkanski, Solid State Commun. 114, 395 (2000).

${ }^{22}$ J.-P. Salvetat, G. A. Briggs, J.-M. Banard, R. R. Bacsa, A. J. Kulik, T. Stöckli, N. A. Burha, and L. Forró, Phys. Rev. Lett. 82, 944 (1999).

${ }^{23}$ M. Cadek, J. N. Coleman, K. P. Rya, V. Nicolosi, G. Bister, A. Fonseca, J. B. Nagy, K. Szostak, F. Beguin, and W. J. Blau, Nano Lett. 4, 353 (2004).

${ }^{24}$ J. N. Coleman, M. Cadek, R. Blake, V. Nicolosi, K. P. Ryan, C. Belton, A. Fonseca, J. B. Nagy, Y. K. Gun'ko, and W. J. Blau, Adv. Funct. Mater. 14, 791 (2004). 